\title{
LETTER TO THE EDITOR The basis of a more contagious 501Y.V1 variant of SARS-CoV-2
}

\author{
Cell Research (2021) 31:720-722; https://doi.org/10.1038/s41422-021-00496-8
}

Dear Editor,

The SARS-CoV-2 virus has infected over one hundred million people (COVID-19 patients) and caused more than two million deaths to date. The number of infected people continues to grow quickly, emphasizing the need for rapid use of effective vaccines. Although two mRNA vaccines based on Spike protein (produced by Pfizer-BioNTech and MODERNA) have been approved for emergency use in the US, ${ }^{1,2}$ the increasing Spike variants that have appeared around the world raise concerns about the continued efficacy of the vaccines. ${ }^{3}$ Monoclonal antibodies, developed by Regeneron and Eli Lilly, specifically targeting the native form of Spike have been approved by the FDA for emergency use. ${ }^{4,5}$ An N501Y variant (Y501) of the Spike protein of SARS-CoV-2 (B.1.1.7, 20I/501Y.V1), first emerged in UK and has now spread to the rest of the world. This variant appears to be much more contagious than the original N501 version. ${ }^{3}$ Furthermore, Y501 mutation is also found in a variant (B.1.351, 20H/501Y. V2) from South Africa and a variant (P1, 20J/501Y.V3) from Brazil. ${ }^{3}$ Unfortunately, this mutation is located at the interaction surface between the RBD and human Angiotensin Converting Enzyme 2 (ACE2). ${ }^{6}$ Thus, the Y501 variation present in B.1.1.7, 20I/501Y.V1 might affect the binding ability of the RBD to bind ACE2. We therefore compared the binding affinity of N501 and Y501 RBD for ACE2, uncovering that the affinity for ACE2 of the Y501-RBD was $\sim 10$ fold higher than that of the N501 version. This may account, at least in part, for the greater infectivity of SARS-CoV-2 with this mutation. Structural modeling data showed that Y501-RBD can form an additional aromatic ring-ring interaction and an additional hydrogen bond with ACE2 by comparison with the RBD of the wild type. In spite of this, sera from individuals immunized with the Pfizer-BioNTech vaccine still efficiently block ACE2 binding to Y501-RBD. Furthermore, Bamlanivimab, the recently FDA approved therapeutic antibody drug for treatment of COVID-19 patients ${ }^{4}$ still binds the variant Y501-RBD as efficiently as it binds the N501-RBD, giving hope that treatment with the existing monoclonal antibodies may still help COVID-19 patients.

To investigate the basis for the more highly infectious property of the N501Y variant, we expressed two versions of SARS-CoV-2 RBD, N501-RBD and Y501-RBD (residues 319-541 aa) in 293F cells (Supplementary information, Fig. S1). Purified proteins of both types were subjected to Surface Plasmon Resonance (SPR) binding assays on a Biacore machine to examine their binding affinities for ACE2. The binding affinity between the native form of RBD (N501$\mathrm{RBD}$ ) and ACE2 was $\sim 5.76 \mathrm{nM}$ (Fig. 1a), similar to the $\sim 4.5 \mathrm{nM}$ previously reported. ${ }^{6}$ To our surprise, the binding affinity of the mutated version of the RBD, Y501-RBD, increased dramatically to $\sim 0.566 \mathrm{nM}$ (Fig. 1b). It has been reported that the affinity of the RBD of SARS-CoV-2 for ACE2 is $\sim 7$ fold higher than that of the RBD of SARS-CoV for the same ligand, ACE2. ${ }^{6,7}$ The $\sim 7$ fold increase in binding affinity could be one major reason that allowed SARSCoV-2 to be more infectious than SARS-CoV. ${ }^{8}$ By extension of this argument, the current mutation of N501 to Y501, and consequently its 10 times greater affinity for ACE2, may account for the increased infection rate by this variant in UK. Likewise, it may also account for the increased transmission rate of both the South African (20H/501Y.V2) and Brazil variants (20J/501Y.V3), although their additional $\mathrm{K} 417 \mathrm{~N} / \mathrm{T}$ and $\mathrm{E} 484 \mathrm{~K}$ mutations besides N501Y may also contribute. ${ }^{9}$

A $2.9 \AA$ resolution Cryo-EM structure of a predicted potential variant containing numerous mutations including E484K, Q498R, N501Y bound to ACE2 has been published. ${ }^{10}$ It shows a detailed interaction of the side chain of Y501 of the RBD and Y41 of ACE2. However, the introduction of Q498R, a mutation that does not exist in current variants, might affect the properties of the Y501 interaction with ACE2 since Q498R is quite close to the 501 position. Thus the reported structure could not unequivocally attribute the $\sim 10$ fold increase in affinity of the variant RBD vs N501-RBD for ACE2 to the Y501 change. Here, we used modern molecular modeling programs to make a prediction about the relevant binding sites. Based on the reported structure of the N501-RBD and ACE2, ${ }^{6}$ we generated a Y501-RBD-ACE2 complex in the program COOT and used the docking program HADDock 2.2 server to carry out optimization (Fig. 1c, d; Supplementary Information). In the case of the wild type SARS-CoV-2 Spike, the side chain of N501 within N501-RBD forms a hydrogen bond with Y41 of ACE2 (Fig. 1C). This interaction is markedly changed in the interaction between the Y501-RBD mutant and ACE2 (Fig. 1d). Although the hydrogen bond between N501-RBD and Y41 of ACE2 disappears for the Y501-RBD mutant, two new forms of interactions, are predicted to exist between Y501-RBD and ACE2 (Fig. 1d). First, the Y501 of Y501-RBD forms two new hydrogen bonds with the side chains of D38 and K353 of ACE2 (Fig. 1d). Second, the aromatic ring of $\mathrm{Y} 501$ also has a strong aromatic stacking interaction ( $\pi$ stacking) with the aromatic ring of $Y 41$ of ACE2 (Fig. 1d). It is quite likely that the ring-ring interaction contributes to the much higher binding affinity between Y501RBD and ACE2. Nevertheless, these detailed interactions still need confirmation with a high-resolution structure of Y501-RBD-ACE2 complex.

It has been reported that $\sim 90 \%$ of broadly neutralizing antibodies from COVID-19 patients target the RBD. ${ }^{11}$ Thus, it is important to test if the binding of these broadly neutralizing antibodies is affected by the change of N501-RBD to Y501-RBD. To this end, we tested sera from individuals immunized with the mRNA vaccine from Pfizer-BioNTech. An N501-RBD specific ELISA detected high titers of $\lg$ antibodies in five immunized individuals (Supplementary information, Fig. S2), suggesting that the vaccine triggered strong immune responses in these individuals. To test the blocking property of broadly neutralizing antibodies toward both native and mutated RBDs, each type of RBD was immobilized on a separate channel of the same Biacore Chip at equal amount. Equal amounts of serum from one vaccinated individual were injected into the two channels. The results showed that both RBDs retained the same amount of antibodies at the different dilutions of injected antibodies

Received: 31 January 2021 Accepted: 1 March 2021

Published online: 23 April 2021 

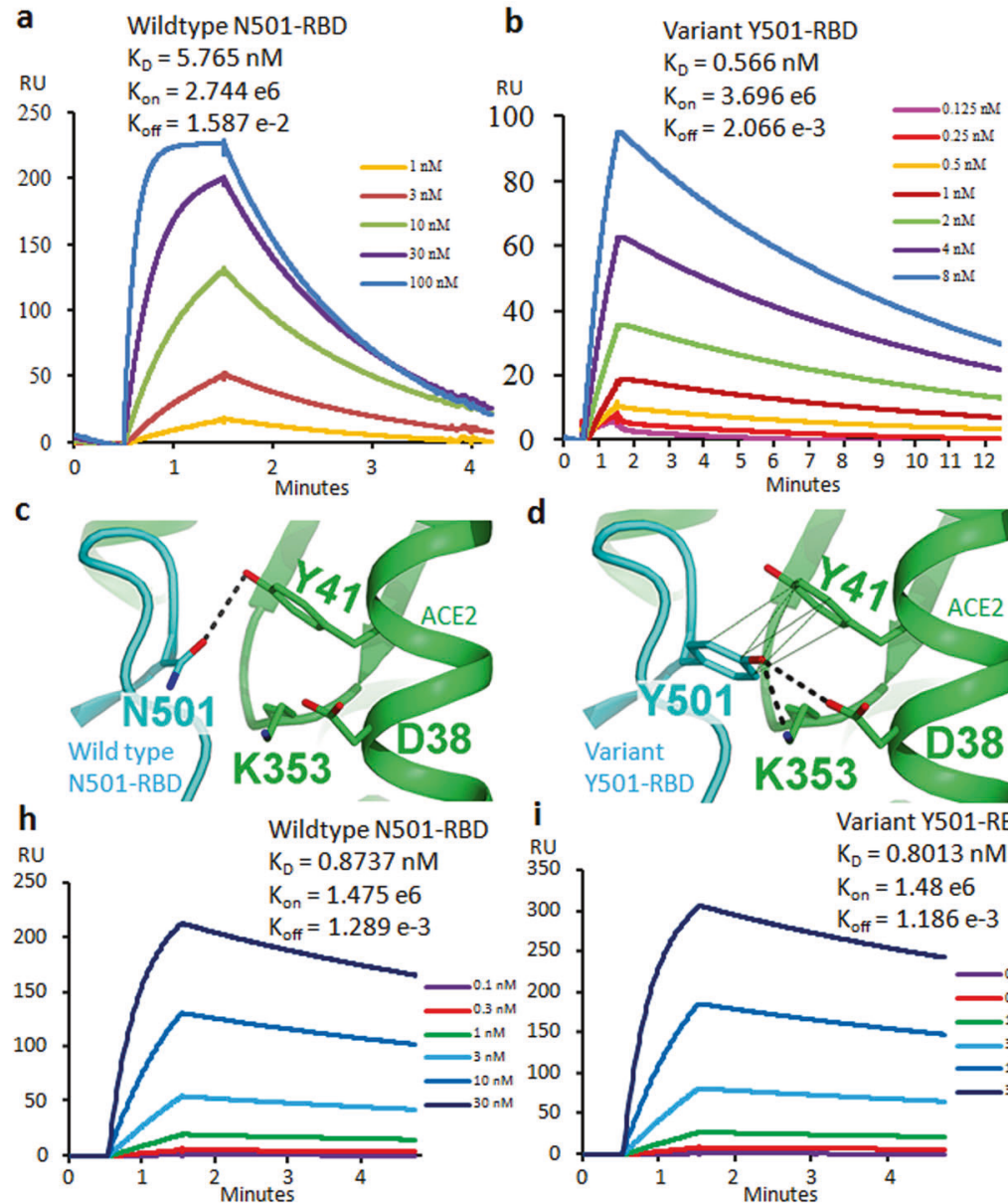

d
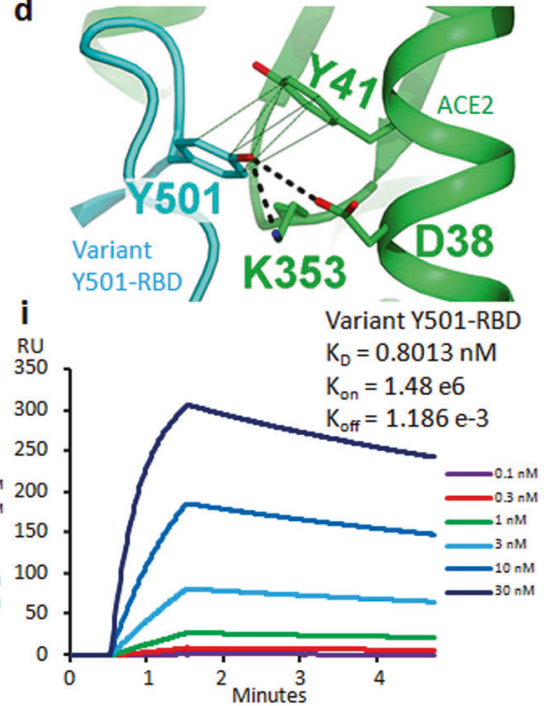

e

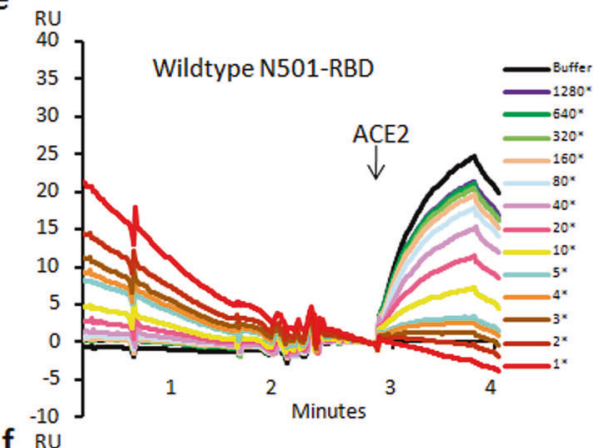

f RU

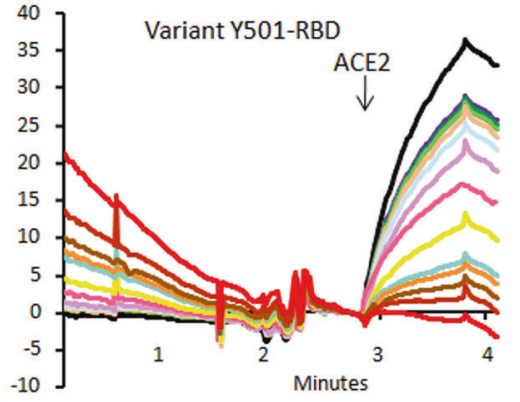

g RU

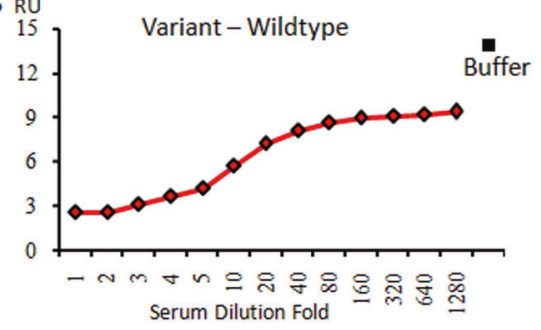

Fig. 1 The UK variant Y501-RBD binds to ACE2 with higher affinity, but remains similar bindings to Bamlanivimab and sera from mRNAvaccinated individuals. a The affinity measurement of N501-RBD to ACE2 by SPR. $K_{D}$, dissociation constant; $K_{\text {on, }}$ association rate; $K_{\text {off }}$ dissociation rate; RU, response unit. b The affinity measurement of Y501-RBD to ACE2 by SPR. c Protein structure of N501-RBD and ACE2. d Protein structure model of Y501-RBD and ACE2. The new hydrogen bonds are shown as heavy dash lines, and the aromatic stacking interaction is shown as light dash lines. e Blocking of ACE2 binding to N501-RBD by different dilutions of mRNA-vaccinated serum. $\mathbf{f}$ Blocking of ACE2 binding to Y501-RBD by different dilutions of mRNA-vaccinated serum. Note that equal amount of N501-RBD and Y501-RBD was coated on two separate channels on the same CM5 chip. Same amount of serum as well as ACE2 were applied in the following dilution steps. Similar amounts of antibodies were retained on both channels (Supplementary information, Fig. S3). g. The difference of ACE2 retained on N501-RBD and Y501-RBD treated with serial dilutions of the serum at $5 \mathrm{~s}$ after ACE2 injection. Each dot value was calculated as the ACE2 binding on Y501-RBD minus ACE2 on N501-RBD. The value of Buffer is the difference of ACE2 on two channels without treatment of the serum. $\mathbf{h}$ The affinity measurement of N501-RBD to Bamlanivimab by SPR. $\mathbf{i}$ The affinity measurement of Y501-RBD to Bamlanivimab by SPR.

(Supplementary information, Fig. S3), suggesting that the N501Y mutation did not affect the binding of antibodies from vaccinated individuals. The serum also blocked binding of ACE2 to both N501-RBD and Y501-RBD (Fig. 1e, f). There was no ACE2 binding for RBDs treated with undiluted serum ( 1 * in Fig. $1 \mathrm{e}, \mathrm{f})$ and ACE2 barely bound the RBDs when the serum was diluted 2-5 times $\left(2^{*}-5^{*}\right.$ in Fig. 1, f). We obtained a similar binding profile from a second individual immunized with the Pfizer-BioNTech vaccine (Supplementary information, Fig. S4). Due to the higher binding affinity of ACE2 to Y501-RBD than N501-RBD, the Y501-RBD channel retains more ACE2 (due to much slower off rate) than that of N501-RBD (Fig. 1g). Altogether, these binding data suggest that the vaccine from the Pfizer-BioNTech could protect people from both versions of SARS-CoV-2.

In Nov 2020, three therapeutic antibodies specific for the RBD of SARS-CoV-2, Bamlanivimab (LY-CoV555) from Eli Lilly, Casirivimab (REGN10933) and Imdevimab (REGN10987) from Regeneron, were authorized for emergency use by the US FDA. ${ }^{5}$ It is important to know whether Y501-RBD is still efficiently blocked or neutralized by these antibodies. Bamlanivimab was derived from a B cell from a convalescent patient which efficiently blocked the binding of N501-RBD to ACE2. ${ }^{12}$ From the structural information, Bamlanivimab binds to a region on RBD close to Y501. ${ }^{13}$ To find out whether Bamlanivimab would also block binding of Y501-RBD to ACE2, we collected the residual Bamlanivimab after the infusion of COVID19 patients and carried out binding assays of this antibody to either N501-RBD or Y501-RBD. To our delight, this antibody bound to N501-RBD or the variant Y501-RBD with a very similar binding affinity of $0.8737 \mathrm{nM}$ (Fig. 1h) and $0.8013 \mathrm{nM}$ (Fig. 1i) respectively. Thus, our data suggest that Bamlanivimab should be still effective in COVID-19 patients infected by the 501Y.V1 variant. The detailed interaction between RBD and Bamlanivimab has been reported. ${ }^{13}$ The N501Y mutation on RBD is close to, but not within, the binding site of Bamlanivimab (Supplementary information, Fig. S5). Of note, the binding affinity between the antibody and the UK variant Y501-RBD $(0.8013 \mathrm{nM})$ is lower than that of Y501-RBD for ACE2 $(0.566 \mathrm{nM})$. This indicates that much higher concentrations of Bamlanivimab might be needed to effectively treat COVID-19 
patients with the UK variant compared to those with wild type. However, variants from South Africa and Brazil have two additional mutations (K417N/T and E484K) within RBD while the side chain of E484 of RBD and the side chain of an Arg (R50) from Bamlanivimab (LY-CoV555) were found to form a salt bridge in the complex structure. ${ }^{13}$ Thus the change of E484 to K484 (in the variants) could lead to a dramatic reduction or loss of interaction by the antibody for the variants. Our follow-up experiments do show that the E484K mutation abolishes the binding between Bamlanivimab and RBD in vitro. ${ }^{14}$ Thus, this monoclonal may not be effective in treatment of COVID-19 patients with either South Africa or Brazilian variant.

The COVID-19 pandemic has become one of the major disasters in human history, but effective means to contain it are still limited. Any mutation associated with an increased rate of infection could be a warning sign of potential escape of the virus and pose challenges to both the available vaccines and therapeutic antibodies. Our data showed that a single mutation of N501Y within RBD gains $\sim 10$ times higher binding affinity, which could account for the much higher infectious rate of the UK variant and possible similar effects for variants from South Africa and Brazil containing the same mutation. Interestingly, this mutation neither affects the binding of sera from vaccinated individuals (Supplementary information, Fig. S4), which contain broadly neutralizing antibodies mostly against RBD, nor changes the association property with a therapeutic antibody, Bamlanivimab. On the other hand, the protection from broadly neutralizing antibodies generated from B cells is just one arm of adaptive immunity. T cells including cytotoxic and the helper T cells compose another arm of protection through recognition of conserved linear peptides derived from SARS-CoV-2 proteins bound to major histocompatibility complex proteins. Many peptides from the Spike protein are likely to be recognized by $\mathrm{T}$ cells, and $\mathrm{T}$ cell responses induced by prior infections or vaccination are unlikely to be broadly reduced by a few amino acid changes in Spike. As elegantly demonstrated by Dr. Alessandro Sette and his collegues, ${ }^{15}$ the $\mathrm{T}$ cell arm of adaptive immunity is effectively induced by infection. Thus SARS-CoV-2-specific T cells may contribute to the low hospitalization rates of vaccinated people infected with the SARS-CoV-2 variants.

\section{ACKNOWLEDGEMENTS}

We thank National Jewish Health for supports. H.L. is partially supported by NIH Grant (GM135421 to G.Z.) and NB Life Laboratory LLC. We thank Biobank Committee of National Jewish Health for approval and Shelley Peterson for contribution. We also thank the Colorado Department of Public Health and Environment (CDPHE) for authorization.

\section{AUTHOR CONTRIBUTIONS}

H.L., P.M., and G.Z. for designing; H.L. and Q.Z. for main experiments; P.W., Z.C., K.A., J.Y., W.D., G.P.D., L.R., and S.K.F. for some experiments; C.J., B.L., and J.K. for some data analysis; H.L., Q.Z., P.M., and G.Z. for final data analysis and writing up.

\section{ADDITIONAL INFORMATION}

Supplementary information The online version contains supplementary material available at https://doi.org/10.1038/s41422-021-00496-8.

Competing interests: H.L. is partially supported by NB Life Laboratory LLC and G.Z. holds equity at NB Life Laboratory LLC.

Haolin Liu ${ }^{1,2}$, Qianqian Zhang ${ }^{3}$, Pengcheng Wei ${ }^{1,2}$, Zhongzhou Chen ${ }^{3}$, Katja Aviszus ${ }^{1,2}$, John Yang ${ }^{4}$, Walter Downing ${ }^{5}$, Chengyu Jiang ${ }^{6}$, Bo Liang ${ }^{7}$, Lyndon Reynoso ${ }^{8}$, Gregory P. Downey ${ }^{4}$, Stephen K. Frankel ${ }^{4}$, John Kappler ${ }^{1,2^{\prime}}$ Philippa Marrack ${ }^{1,2}$ and Gongyi Zhang ${ }^{1,2}$

${ }^{1}$ Department of Immunology and Genomic Medicine, National Jewish Health, Denver, CO 80206, USA; ${ }^{2}$ Department of Immunology and Microbiology, School of Medicine, Anschutz Medical Center, University of Colorado, Aurora, CO 80216, USA; ${ }^{3}$ State Key Laboratory of Agrobiotechnology, College of Biological Sciences, China

Agriculture University, Beijing 100193, China; ${ }^{4}$ Department of Medicine, National Jewish Health, Denver, CO 80206, USA; ${ }^{5}$ Department of Nursing, National Jewish Health, Denver, CO 80206, USA; ${ }^{6}$ Chinese Academy of Medical Sciences, Department of Biochemistry and Molecular Biology, Peking Union Medical College, Tsinghua University, Beijing 100005, China; ${ }^{7}$ Department of Biochemistry, Emory University of School of Medicine, Atlanta, GA 30322, USA and ${ }^{8}$ Department of Pharmacy, National Jewish Health, Denver, CO 80206, USA

These authors contributed equally: Haolin Liu, Qianqian Zhang Correspondence: Haolin Liu (Liuh@NJhealth.org) or Philippa Marrack (Marrackp@NJhealth.org) or Gongyi Zhang (Zhangg@NJhealth.org)

\section{REFERENCES}

1. U. S. Food \& Drug. Pfizer-BioNTech vaccine. https://www.fda.gov/emergencypreparedness-and-response/coronavirus-disease-2019-covid-19/pfizer-biontechcovid-19-vaccine (2020).

2. U. S. Food \& Drug. Moderna vaccine. https://www.fda.gov/emergencypreparedness-and-response/coronavirus-disease-2019-covid-19/moderna-covid19-vaccine (2020).

3. CDC. SARS-CoV-2 variants. https://www.cdc.gov/coronavirus/2019-ncov/more/ science-and-research/scientific-brief-emerging-variants.html (2021).

4. U. S. Food \& Drug. Bamlanivimab. https://www.fda.gov/news-events/pressannouncements/coronavirus-covid-19-update-fda-authorizes-monoclonalantibody-treatment-covid-19 (2020).

5. U. S. Food \& Drug. Casirivimab \& imdevimab. https://www.fda.gov/news-events/ press-announcements/coronavirus-covid-19-update-fda-authorizes-monoclonalantibodies-treatment-covid-19 (2020).

6. Lan, J. et al. Nature 581, 215-220 (2020).

7. Wrapp, D. et al. Science 367, 1260-1263 (2020)

8. Starr, T. N. et al. Cell 182, 1295-1310 (2020).

9. Tegally, H. et al. medRxiv https://doi.org/10.1101/2020.12.21.20248640 (2020).

10. Zahradnik, et al. bioRxiv https://doi.org/10.1101/2021.01.06.425392 (2021).

11. Piccoli, L. et al. Cell 183, 1024-1042 (2020).

12. Chen, P. et al. N. Engl. J. Med. 384, 229-237 (2021).

13. Jones, B. E. bioRxiv https://doi.org/10.1101/2020.09.30.318972 (2020)

14. Liu, H. bioRxiv https://doi.org/10.1101/2021.02.16.431305 (2021).

15. Grifoni, A. et al. Cell 181, 1489-1501 (2020). 